\title{
El contexto en la enseñanza de la química: Análisis de secuencias de enseñanza y aprendizaje diseñadas por profesores de ciencias de secundaria en formación inicial
}

\author{
Sylvia Moraga Toledo \\ Facultad de Ciencias de la Educación. Universidad de Playa Ancha, Valparaíso, Chile. \\ Sylvia_moraga@upla.cl \\ ORCID: https:// orcid.org/0000-0002-7985-6842
}

\author{
Mariona Espinet Blanch \\ Departamento de Didáctica de la Matemática y de las Ciencias Experimentales, Universidad Autónoma de \\ Barcelona, Catalunya, España.mariona.espinet@uab.cat \\ ORCID: https:/ / orcid.org/0000-0002-6072-1497
}

\section{Cristian Merino Rubilar}

Instituto de Química. Pontificia Universidad Católica de Valparaíso, Chile.cristian.merino@pucu.cl ORCID: https:// orcid.org/0000-0002-1156-2581

[Recibido: 3 Junio 2018. Revisado: 2 Agosto 2018. Aceptado: 19 Noviembre 2018]

Resumen: Este trabajo pretende identificar las dificultades que tienen los estudiantes del Máster de Formación del Profesorado de Secundaria (MFPS) de Ciencias de las cinco universidades públicas catalanas en el diseño de secuencias de enseñanza y aprendizaje (SEA) de química contextualizadas. La pregunta que guía la investigación es: ¿Qué función cumplen los contextos en las SEA de química diseñados por los profesores de ciencias de secundaria en formación inicial? La investigación propone el análisis de contenido de 5 SEA diseñadas por los profesores en formación inicial durante el curso 2015-16 con respecto a las características de los contextos utilizados a lo largo de la SEA a través de la aplicación de la Rúbrica de Indicadores de Contexto (RIC) creada. Se han identificado tres perfiles de contextualización en base a cinco indicadores de contextualización: autenticidad, relevancia, persistencia, indagación y construcción. En los tres perfiles se constatan las dificultades para introducir contextos que sean persistentes a lo largo de la SEA y que faciliten la indagación científica.

Palabras claves: Educación científica basado en contextos (EBC), Formación inicial del profesorado, Diseño de secuencias de enseñanza y aprendizaje, enseñanza de la química, contexto y concepto.

Context in chemistry education: analysis of pre-service secondary science teacher's teaching and learning sequences.

Abstract: The research presented aims at identifying the difficulties pre-service secondary science teachers have
in designing context-based chemistry teaching and learning sequences (TLS). The question guiding this study is
the following: What function do contexts perform within chemistry TLS designed by pre-service secondary
science teachers? A content analysis of five chemistry TLS written in $2015-16$ by five pre-service secondary
science teachers from the master program offered by the five public universities in the community of Catalonia,
Spain was conducted. The focus of the analysis dealt with the characteristics of contexts used in TLS using the
Rubric of Context Indicators (RIC) created for this purpose. Three TLS contextual profiles have been identified
using the 5 RIC context indicators such as authenticity, relevance, persistence, inquiry and construction. All pre-
service secondary science teachers from the three contextual profiles have written chemistry TSL showing
difficulties in the use of contexts that are persistent along the TLS and promote scientific inquiry.

Keywords: Pre-service secondary science teacher education, teaching -learning sequences, context based learning, chemistry education, context and concept.

Para citar este artículo: Moraga, S., Espinet, M., Merino, C. (2019) El contexto en la enseñanza de la química: análisis de secuencias de enseñanza y aprendizaje diseñadas por profesores de ciencias en formación inicial. Revista Eureka sobre Enseñanza y Divulgación de las Ciencias 16(1), $1604 . \quad$ doi: 10.25267/Rev_Eureka_ensen_divulg_cienc.2019.v16.11.1604 


\section{Introducción}

La investigación que aquí se presenta se sitúa en el campo de la Enseñanza de las Ciencias Basada en Contexto (EBC), y más concretamente en la formación del profesorado de ciencias para enseñar una química basada en contexto. Sus objetivos emergen de la reflexión acerca de cómo el profesor de ciencias diseña sus clases de manera que permitan al estudiante pensar científicamente desarrollando, evaluando y revisando modelos, explicaciones y teorías de la química en el marco de una ciencia escolar (Izquierdo, 2006). Diseñar SEA de química contextualizadas que faciliten la construcción de modelos teóricos escolares, resulta un desafío importante tanto para el profesorado en activo como el de formación inicial. Desde esta perspectiva emerge la importancia de profundizar y evidenciar el proceso del docente cuando diseña su clase en contexto. El presente estudio se plantea la siguiente pregunta central: ¿Qué función cumplen los contextos en las SEA de química diseñados por los profesores de ciencias de secundaria en formación inicial? Pretendemos con ello realizar una aportación a la formación del profesorado de ciencias de educación secundaria a través de la identificación de dificultades que tienen los futuros profesores al diseñar SEA de química contextualizadas.

\section{Marco de referencia}

\section{Enseñanza basada en el contexto (EBC)}

Se entiende la EBC como una metodología consistente en construir y desarrollar conocimientos científicos a partir de una situación del mundo real. Esta situación se usa como estructura central para ir introduciendo los conceptos científicos a medida que son necesarios y desarrollar así, una mejor comprensión de la situación planteada (King y Richtie, 2012). La EBC toma un contexto como punto de partida para introducir un tema y activar la curiosidad de los estudiantes, logrando mejorar el interés, la motivación y la actitud hacia la Ciencia. Este enfoque promueve un aprendizaje más significativo de las ideas científicas, ya que facilita las conexiones teoría-realidad, y atenúa la separación entre ambas, propia de la enseñanza tradicional. Diversas son las investigaciones en Didáctica de las Ciencias que apuntan los beneficios de una enseñanza de las ciencias basada en contexto (Pilot y Bulte, 2006; Broman y Parchmann, 2014). En concreto, las investigaciones y reflexiones realizadas por el grupo Lenguaje y Enseñanza de las Ciencias (LIEC) apuntan al potencial de utilizar el contexto en la Enseñanza de las Ciencias e indican que posibilita (Sanmartí y Márquez 2017):

a) Reconocer la utilidad del conocimiento aprendido (tanto científica como en relación con la acción). Construir conocimiento científico con sentido y transferible.

b) Generar una actividad científica escolar (indagar, argumentar y modelizar).

c) Estimular la necesidad de aprender y de seguir aprendiendo.

d) Generar emociones positivas en el alumnado al descubrir retos que le llevan a formular preguntas estimulantes, implicarse en la búsqueda de soluciones, y experimentar la satisfacción de encontrarlas.

e) Implicarse en acciones que repercuten en la comunidad (escolar, del entorno próximo o global).

Resulta necesario realizar un esfuerzo en la formación inicial y permanente del profesorado para, por un lado, difundir los principios y modelos de la EBC y por otro, que abarquen una población más amplia de docentes de ciencias. 


\section{La función del contexto en la EBC}

En el marco de esta investigación entendemos por contexto el escenario que permite generar en el alumnado la necesidad de entender alguno de los fenómenos que ocurren en el mundo. Su comprensión, a través de la construcción y uso de unas ideas científicas y sobre la Ciencia, debería capacitar a los aprendices para actuar en el marco de una comunidad de práctica (Sanmartí y Márquez 2017). Para ello es necesario la adquisición y uso de unas ideas organizadas alrededor de modelos científicos y sobre la ciencia como actividad humana. Los escenarios que constituyen los contextos favorecen la emisión de cuestiones que tienen una relevancia para el alumnado y le permiten conectar con su realidad. Existe una diversidad de funciones del contexto en los procesos de Enseñanza y Aprendizaje de las Ciencias. Gilbert (2006) y Gilbert et al. (2011) identificaron cuatro modelos de contextos de acuerdo con la relación que mantienen el contexto y la construcción de conocimiento científico en una SEA de educación científica: En el 1er modelo, el contexto se utiliza como la aplicación directa de los conceptos, como reciprocidad entre los conceptos y aplicaciones.; en el $2^{\circ}$ modelo, el contexto se utiliza como un vehículo para relacionar los conceptos con sus aplicaciones. Durante la enseñanza de estos conceptos la relación con el contexto se supone que es cíclica, lo que hace de este modelo un poco más complejo en comparación con el primer modelo; en el 3er. modelo los conceptos científicos se vinculan a modelos mentales que se utilizan para interpretar las situaciones planteadas, aun cuando la existencia de modelos previos puede interferir en este proceso; y en el $4^{\circ}$ modelo, entendido como el más adecuado para el desarrollo de enfoques contextualizados, se reconoce el contexto como una entidad cultural inmersa en la sociedad, de forma que el aprendizaje ocurre dentro del proceso de interacción entre los alumnos y el profesor, a través de temas que se consideran importantes para la vida comunitaria en sociedad.

En esta misma línea, De Jong (2008) identificó tres modelos de contexto para diseñar una SEA. El modelo tradicional se utiliza para ilustrar un concepto abstracto o puede ser utilizado por el alumno para aplicar sus conocimientos de un determinado concepto. Usa el contexto solo por razones decorativas y se compara con el primer modelo de Gilbert et al, (2011). El modelo moderno comienza con un contexto que tiene un propósito orientador y motivador hacia el estudiante permitiéndole aprender nuevos conceptos. Finalmente, el modelo reciente combina todas las funciones de los anteriores: se inicia con un contexto, pero después los conceptos se introducen en nuevos contextos con el fin de denotar sus aplicaciones.

Tanto De Jong como Gilbert ponen de manifiesto la función del contexto en la construcción de conocimiento conceptual en una propuesta de EBC. Los contextos utilizados en la enseñanza de la química son de diferente naturaleza y están situados en momentos diferentes de las SEA. Entendemos, por tanto, que la función del contexto es clave y debe ser abordada en la formación del profesorado de ciencias de educación secundaria durante el diseño de SEA de química. Coincidiendo con Lupión, López y Blanco (2017) señalan que es importante capacitar a los maestros para que desarrollen, implementen y evalúen los resultados de sus propias propuestas ya que constituye una buena forma de proporcionarles la experiencia práctica de este enfoque y de transferir el conocimiento al aula.

\section{El diseño de SEA en la formación inicial del profesorado de secundaria de ciencias}

La elaboración de una SEA se concibe como una oportunidad valiosa para la formación del profesorado de ciencias ya que promueve la dialéctica teoría-práctica durante el diseño y la planificación didáctica (Astudillo et al. 2011). En esta trayectoria el docente desarrolla procesos reflexivos y recursivos que le permiten la construcción de un conocimiento práctico cada vez más complejo. La investigación sobre el diseño de SEA por parte del profesorado de ciencias empezó en los años ochenta (Mehéut y Psillos, 2004). Los resultados de estas investigaciones 
se centraron fundamentalmente en el aprendizaje del estudiante y dejaron en un segundo plano o directamente no consideraron otros dos elementos fundamentales de una situación de aprendizaje: el conocimiento que se debe desarrollar y el papel de los docentes (Arriassecq, et al. 2017; Mehuet, 2005). Investigaciones más recientes se han focalizado en las dificultades que tienen los docentes de ciencias de formación inicial en el diseño de SEA. Destacamos algunas de estas investigaciones que tienen relación con el uso de contextos en el diseño de SEA de ciencias. Bogdan Toma, Greca y Meneses-Villagrá (2017) muestran que los estudiantes que diseñan SEA indagadoras tienden a concebir la indagación como un quehacer práctico en el laboratorio totalmente descontextualizado en la mayoría de las ocasiones. Finalmente destacamos el trabajo de De Jong (2008) que aborda el análisis de SEA elaboradas por los profesores en formación inicial. Este autor apunta que una de las dificultades más importante del profesorado en la EBC reside en la defectuosa conexión entre el contexto y los conceptos enseñados. Concluimos que el uso del contexto en el diseño de una SEA constituye una dificultad destacada por parte del profesorado de ciencias en formación inicial tanto si se trata de una SEA bajo una orientación de EBC, como de modelización o de indagación. Esta dificultad se puede articular alrededor de la tensión entre el uso de contextos y la construcción de modelos que permitan a los estudiantes comprender los fenómenos que emergen en situaciones relevantes.

\section{Metodología}

\section{El contexto y selección de la muestra}

El contexto para la recogida de datos de la presente investigación lo constituye el Máster de formación del profesorado de educación secundaria (MFPS) de Ciencias de Catalunya. Este máster es de orientación profesional imprescindible para ejercer la docencia en la educación secundaria obligatoria, bachillerato y la formación profesional que sustituye el antiguo certificado de aptitud pedagógica (CAP). Las universidades participantes están constituidas por las cinco Universidades que imparten el MFPS de Ciencias durante el curso 2015-16 en la Catalunya: (1) Universidad Autónoma de Barcelona, (2) Universidad de Barcelona, (3) Universidad de Girona, (4) Universidad Pompeu Fabra, y (5) Universidad de Rovira y Virgili.

Se recogieron 20 SEA de educación química diseñadas por los profesores en formación inicial distribuidas tal como se muestra en la Tabla 1. De las SEA recogidas se seleccionaron, para el presente estudio, una de cada universidad de acuerdo con los siguientes criterios: (a) existencia de una estructura en el diseño de la SEA y (b) presencia explícita de las actividades que desarrollará el estudiante. Las universidades se han identificado con una letra para resguardar su identidad.

Tabla 1. Características generales de las SEA en el período 2015-16

\begin{tabular}{|c|c|c|c|c|c|}
\hline Institución & $\mathbf{A}$ & $\mathbf{B}$ & $\mathbf{C}$ & $\mathbf{D}$ & $\mathbf{E}$ \\
\hline $\begin{array}{c}\mathbf{N}^{\mathbf{o}} \text { de SEA } \\
\text { analizadas/ total }\end{array}$ & $1 / 1$ & $1 / 6$ & $1 / 3$ & $1 / 5$ & $1 / 6$ \\
\hline Nivel Educativo & $3^{\circ} \mathrm{ESO}$ & $3^{\circ} \mathrm{ESO}$ & $3^{\circ} \mathrm{ESO}$ & $1^{\circ} \mathrm{ESO}$ & $2^{\circ}$ Bachillerato \\
\hline Tema & La materia & $\begin{array}{c}\text { Reacciones } \\
\text { químicas }\end{array}$ & $\begin{array}{c}\text { Reacciones } \\
\text { químicas }\end{array}$ & $\begin{array}{c}\text { Clasificación } \\
\text { de la materia }\end{array}$ & Disoluciones \\
\hline
\end{tabular}

\section{Enfoque metodológico}

La presente investigación se enmarca en un paradigma cualitativo basado en el análisis de textos (SEA). Se focaliza en analizar las SEA que diseñan los profesores en formación inicial y no en el proceso de enseñanza que tuvieron los profesores en formación inicial para su 
construcción, por lo tanto, utiliza un enfoque metodológico del análisis del contenido (Weber, 1990) para el estudio de las SEA de química diseñadas por los profesores de ciencias de educación secundaria en formación inicial. A través de un proceso de segmentación progresiva y de la construcción de un sistema de categorías inductivo-deductivo se procede a la asignación de significado en base a las actividades de la SEA como unidad de análisis.

\section{Segmentación de los datos}

Para realizar el análisis de las SEA de química se procede a segmentarlas en tres dimensiones: estructura, actividad y contexto. La segmentación estructural de cada SEA se realiza a partir de la asignación de tres fases: inicio, medio y final. La fase de inicio se sitúa normalmente al principio de la SEA e incluye aquellas actividades destinadas a presentar la temática de estudio, explorar las ideas previas del alumnado, y presentar los objetivos de la SEA. La fase del medio está constituida por todas aquellas actividades destinadas a desarrollar por parte del alumnado las competencias identificadas en los objetivos de la SEA. Por último, la fase del final la conforman todas aquellas actividades que pretenden la aplicación de las competencias adquiridas por el alumnado en la fase anterior y cerrar así la SEA. La segmentación por actividades se caracteriza por la identificación del número total de actividades que constituyen cada segmento estructural de la SEA y por la delimitación de los segmentos textuales de la SEA asociados a cada actividad. Finalmente, la segmentación por contexto supone la identificación de aquellos fragmentos textuales asociados a un contexto dentro de cada una de las actividades identificadas en la segmentación por actividad. Concluimos de este modo, que los contextos están presentes en las actividades de una SEA y están materializadas en los textos multimodales.

\section{Construcción de categorías}

Para identificar la función que tienen los contextos en las SEA de química se ha utilizado una adaptación de los cinco indicadores de contexto propuestos por el Grupo Lenguaje y Enseñanza de las Ciencias (LIEC) ${ }^{1}$ : autenticidad, relevancia, persistencia, indagación y construcción (Tabla 2). A partir de estos indicadores se ha diseñado una rúbrica que permite identificar el grado de presencia del indicador en cada actividad de la SEA. Para ello, se dividió cada indicador en tres criterios: insatisfactorio, moderadamente satisfactorio y satisfactorio y a la vez se ha identificado cada uno de ellos con un código, 1, 2 y 3 respectivamente (Tabla 2). A continuación, procedemos a una descripción de cada uno de los cinco indicadores de contexto mostrados en la RIC de la Tabla 2.

\section{- Autenticidad de los contextos de una SEA}

Se entiende por autenticidad de un contexto en el marco de una SEA a una situación real o verosímil que genera un problema que puede tener sentido o interés para el estudiante. Autores como Prins et al. (2008) y Bulte et al. (2006) han trabajado con el concepto de práctica auténtica descrita como un grupo homogéneo de personas que forman una "comunidad de práctica", unidos por un propósito común de resolver un problema práctico específico relacionado con la Ciencia/Tecnología y con un procedimiento común que conduce a su solución. El hecho de tener que resolver un problema que parte de una situación real, obliga a los estudiantes a contextualizar, es decir a transformar de manera progresiva conceptos, enunciados o conocimientos teóricos en decisiones y acciones prácticas en la realización de una actividad (Jiménez-Aleixandre y Reigosa 2006). Por tanto, y de acuerdo con Marchant y Sanmartí (2014), en las prácticas auténticas se parte de la premisa que los conceptos científicos ya han sido construidos previamente (al menos en una primera aproximación y probablemente a través de una enseñanza descontextualizada) y la práctica auténtica sirve para poner en

\footnotetext{
www.cienciescontext.com
} 
práctica y desarrollar estos modelos en una situación real con la finalidad de que lleguen a ser significativos para la persona que aprende.

\section{- Relevancia de los contextos de una SEA}

Se entiende por relevancia de un contexto en el marco de una SEA, el nivel de conectividad e implicaciones a nivel personal, social o vocacional que se pueden establecer a partir de la situación planteada. Consideramos que una situación que aporte más de una dimensión generará una visión más integral en el alumno permitiéndole tener un mayor número de conectividad a partir de un fenómeno, por lo tanto, la relevancia del contexto será mayor.

De acuerdo con Parchmann et al. (2006), la educación debe preparar a la generación joven para convertirse en ciudadanos críticos y responsables, pudiendo contribuir al desarrollo de la sociedad. Sugiere para ello, y siguiendo las aportaciones de la revisión realizada por Stuckey, Hofstein, Mamlok-Naaman y Eilks (2013) tres dimensiones de relevancia de la educación científica, que abarca componentes tanto intrínsecos como extrínsecos. Las dimensiones intrínsecas abarcan los intereses y motivos de los estudiantes; la dimensión extrínseca cubre las expectativas éticamente justificadas del entorno personal y de la sociedad en la que operan y viven. Estas dimensiones son:

a) Personal: Se relaciona con las situaciones de la vida cotidiana, promueve la curiosidad y el interés en los estudiantes y les proporciona las habilidades necesarias para su vida actual y futura.

b) Social: Se centra en la preparación de los alumnos para la autodeterminación y responsabilidad de la vida en la sociedad mediante la comprensión de la interdependencia y la interacción de la ciencia y la sociedad, desarrollando habilidades para la participación, y competencias para contribuir al desarrollo sostenible de la sociedad.

c) Vocacional: Se dispone a ofrecer una orientación para futuras profesiones, incluyendo la formación profesional o académica y la apertura de oportunidades de ingreso en una carrera

Estas dimensiones se pueden superponer y muchos aspectos de los fenómenos que se trabajan pueden contribuir a más de una dimensión con respecto a cómo se interpretan y ejecutan. Por ejemplo, la orientación profesional puede ser parte de la relevancia vocacional, pero también puede vincularse a la curiosidad personal o responder a la demanda de más científicos, lo que permite la prosperidad para el futuro de la sociedad (Stuckey et al., 2013).

\section{- Persistencia de los contextos de una SEA}

Se entiende por persistencia de un contexto en el marco de una SEA a la existencia de textos que materializan el contexto a lo largo de varias actividades de enseñanza-aprendizaje de una SEA. Existe un debate en la comunidad de la Didáctica de las Ciencias sobre si una buena SEA debe articularse alrededor de uno o varios contextos diferentes. A raíz de este debate hemos encontrado, por una parte, un estudio realizado por Marchan y Sanmartí, (2015) en el que señalan que la diversidad de contextos alrededor de un mismo modelo teórico puede contribuir a que el estudiante tome conciencia de la universalidad y utilidad de las teorías de la Ciencia, posibilitando su transferencia. Por lo tanto, es necesario que cada contexto y subcontexto no sea un mero pretexto, sino que se constituya en hilo conductor para introducir y construir ideas claves. En la misma línea, Podschuweit y Bernholt (2018), indican que es prometedor involucrar a los estudiantes a entornos de aprendizaje que se contextualizan de forma heterogénea (que abarca una amplia gama de contextos), ya que los contextos más heterogéneos deberían implicar una variedad de nuevos elementos causales favoreciendo la transferencia de los conceptos. Y por otro lado, nos hemos encontrado con investigaciones de Rodríguez-Mora y Blanco (2015); Blanco, Franco-Mariscal y España, (2015) en donde el 
contexto elegido juega un papel fundamental en las distintas etapas que conforman el proceso de enseñanza, y las situaciones y problemas procedentes del contexto pasan a construir el eje central que guía y estructura toda la secuencia (Blanco, España y Rodríguez, 2012). Por lo tanto, si bien estamos de acuerdo con los objetivos que se logran en los alumnos a partir de SEA diseñadas con contexto heterogéneos, pero también es importante que el profesor en formación inicial adquiera en primera instancia las habilidades, las herramientas necesarias para conectar y articular un contexto con sus múltiples funciones a lo largo de las actividades que conforman la SEA.

- Nivel Indagador de los contextos de una SEA

Se entiende por nivel indagador de un contexto en el marco de una SEA, cuando el texto que materializa el contexto propone preguntas que movilice la capacidad de indagar científicamente y con ello nos basamos en el primer significado de indagación de Couso (2014) que señala que una de las capacidades cognitivas que los estudiantes deben desarrollar es la capacidad de indagar o investigar científicamente -generalmente denominadas destrezas indagativas (inquiry skills)- a partir de preguntas investigables, aquellas que requieren relacionar las pruebas con una idea científica clave. En esta línea, un contexto que genere la indagación según Tamir y Garcia (1992) implica desarrollar en los estudiantes un sistema de pensamiento mediante un ejercicio intelectual donde se estimula el plantearse preguntas a través de los fenómenos y desarrollar prácticas científicas. En su artículo, los autores plantean unos niveles de indagación en el trabajo práctico de laboratorio (ILI, The Inquiry Level Index) basados en los diseñados por Herron (1971). El ILI consta de 4 niveles determinados a partir de las tareas de indagación que los estudiantes deben realizar. Nivel cero: Se les da la pregunta, el método y la respuesta. El estudiante debe seguir las instrucciones y obtener los resultados indicados en el texto. Este tipo de práctica de laboratorio se denomina también verificación o confirmación. Nivel uno: Se da la pregunta y el método, y el estudiante tiene que hallar la respuesta. Nivel dos: Se da la pregunta y el estudiante debe encontrar un método y una respuesta. Nivel tres: Se le indica un fenómeno y tiene que formular una pregunta adecuada y encontrar un método y una respuesta a la pregunta.

- Nivel Constructor de los contextos de una SEA

Un contexto es constructor en el marco de una SEA, si el texto que materializa el contexto propone buenas preguntas que, de acuerdo a Roca, Márquez y Sanmartí (2013), de manera implícita pueden mostrar hasta qué punto el conocimiento es compartido o no entre los interlocutores; ello puede favorecer el diálogo, ya que ayuda a avanzar en la elaboración de los conceptos implicados facilitando la reorganización de las relaciones que, implícita o explícitamente, se han manifestado y puesto en evidencia a través de la pregunta. Por lo tanto, un profesor puede emplear las preguntas con diversas finalidades instructivas: para motivar, para centrar la atención, para incrementar la participación del alumno, etc.

Los criterios utilizados para determinar el grado de presencia de cada indicador de un contexto en una actividad de la SEA de química se presentan en la Tabla 2. 
Tabla 2. Rúbrica de los indicadores de contextos (RIC)

\begin{tabular}{|c|c|c|c|}
\hline Indicador & \multicolumn{3}{|c|}{ Criterios } \\
\hline & $\begin{array}{l}\text { Insatisfactorio } \\
\text { (1) }\end{array}$ & $\begin{array}{l}\text { Moderadamente satisfactorio } \\
\text { (2) }\end{array}$ & $\begin{array}{l}\text { Satisfactorio } \\
\text { (3) }\end{array}$ \\
\hline Autenticidad & $\begin{array}{l}\text { El contexto donde se } \\
\text { desarrolla la actividad no } \\
\text { es real o verosímil y no hay } \\
\text { un problema a solucionar. }\end{array}$ & $\begin{array}{l}\text { El contexto donde se desarrolla } \\
\text { la actividad es real o verosímil } \\
\text { pero no hay un problema a } \\
\text { solucionar. }\end{array}$ & $\begin{array}{l}\text { El contexto donde se desarrolla } \\
\text { la actividad está inserto en un } \\
\text { problema real o verosímil y hay } \\
\text { un problema a solucionar. }\end{array}$ \\
\hline Relevancia & $\begin{array}{l}\text { El contexto donde se } \\
\text { desarrolla la actividad no } \\
\text { se relaciona con ninguna } \\
\text { de las dimensiones (social, } \\
\text { personal y vocacional). }\end{array}$ & $\begin{array}{l}\text { El contexto donde se desarrolla } \\
\text { la actividad se relaciona con } \\
\text { alguna de las dimensiones (social, } \\
\text { personal y vocacional). }\end{array}$ & $\begin{array}{l}\text { El contexto donde se desarrolla } \\
\text { la actividad está relacionado al } \\
\text { menos con dos o más de las } \\
\text { dimensiones (social, personal y } \\
\text { vocacional). }\end{array}$ \\
\hline Persistencia & $\begin{array}{l}\text { Los contextos están } \\
\text { presentes en una } \\
\text { actividad de la SEA. }\end{array}$ & $\begin{array}{l}\text { Un mismo contexto está } \\
\text { presente en algunas actividades } \\
\text { de la SEA. }\end{array}$ & $\begin{array}{l}\text { El mismo contexto está presente } \\
\text { en la fase inicial, media y final de } \\
\text { la SEA. }\end{array}$ \\
\hline Indagación & $\begin{array}{l}\text { El contexto no presenta } \\
\text { un trabajo práctico. }\end{array}$ & $\begin{array}{l}\text { El contexto plantea un trabajo } \\
\text { práctico con un nivel de } \\
\text { indagación } 0 \text { y } 1 \text { del ILI. }\end{array}$ & $\begin{array}{l}\text { El contexto plantea un trabajo } \\
\text { práctico con un nivel de } \\
\text { indagación } 2-3 \text { del ILI. }\end{array}$ \\
\hline Construcción & \begin{tabular}{lrrr}
\multicolumn{2}{l}{ Las preguntas } & de & la \\
actividad & no & están \\
relacionadas & con & el \\
contexto. & & &
\end{tabular} & $\begin{array}{l}\text { El contexto donde se desarrolla } \\
\text { la actividad plantea preguntas } \\
\text { cerradas conectadas con el } \\
\text { contexto. }\end{array}$ & $\begin{array}{l}\text { El contexto donde se desarrolla } \\
\text { la actividad presenta preguntas } \\
\text { abiertas conectadas con el } \\
\text { contexto. }\end{array}$ \\
\hline
\end{tabular}

La información obtenida a partir del análisis de las características de los contextos de las 5 SEA se distribuyó en tablas de codificación correspondientes a cada SEA. La Tabla 3 muestra un ejemplo de codificación que corresponde a la SEA "D" titulada "La materia". En ella se incluyen los siguientes aspectos: (a) estructura de la SEA en tres partes: inicio, medio y final; (b) numeración de las actividades que presenta la SEA (A2-A18); (c) identificación y numeración de los contextos presentes en las actividades. Si las actividades presentan los mismos contextos se mantiene la misma enumeración, pero si el contexto es diferente se le asigna un número nuevo. Aquellas actividades que no presentan contextos se les designa como no contextos y se simboliza con la nomenclatura C0; y (d) asignación de un criterio (1, 2 o 3) para cada uno de los cinco indicadores correspondientes a cada una de las actividades de la SEA.

Tabla 3. Codificación de la SEA de química D titulada "La materia"

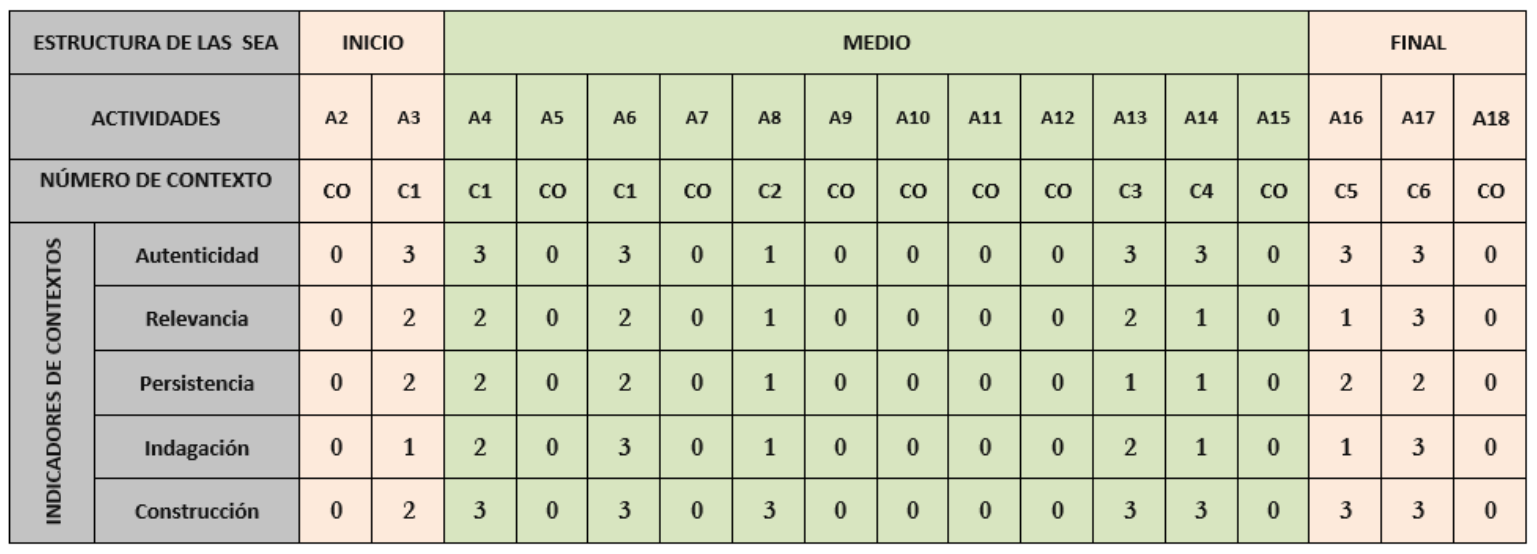




\section{Validación del instrumento de análisis}

El método utilizado para la validación de los instrumentos de análisis fue a través del juicio de expertos, proceso que se realizó en la fase piloto del estudio. Participaron en la validación dos expertas del área de la Didáctica de las Ciencias con una reconocida trayectoria en el campo de la docencia y de la investigación en la Universidad Autónoma de Barcelona (UAB).

Los resultados de este análisis sirvieron para resolver las discrepancias o desacuerdos entre las juezas y la investigadora. De ese modo, se trabajó en la mejora de los ítems que lo requirieron hasta construir un nuevo instrumento más explícito que el anterior y que podría ser utilizado en la siguiente etapa de la investigación que corresponde al estudio principal.

\section{Resultados}

Previo a conocer las características de los contextos de las SEA, es importante conocer las actividades contextualizadas y no contextualizadas que presenta una SEA, para luego aplicar la RIC a aquellas actividades contextualizadas en la SEA. La Tabla 4 muestra el número de actividades contextualizadas y no contextualizadas presentes en las 5 SEA.

Tabla 4. Actividades contextualizadas y no contextualizadas de las SEA

\begin{tabular}{|c|c|c|c|c|c|c|}
\hline UNIVERSIDAD & A & B & $\mathrm{C}$ & $\mathrm{D}$ & $\mathrm{E}$ & TOTAL \\
\hline $\begin{array}{c}\text { Número y } \\
\text { porcentaje } \\
\text { de actividades } \\
\text { por SEA }\end{array}$ & $\begin{array}{l}n=11 \\
(15 \%)\end{array}$ & $\begin{array}{r}n=29 \\
(38 \%)\end{array}$ & $\begin{array}{c}n=9 \\
(12 \%)\end{array}$ & $\begin{array}{c}n=17 \\
(22 \%)\end{array}$ & $\begin{array}{c}n=10 \\
(13 \%)\end{array}$ & $\begin{array}{c}n=76 \\
(100 \%)\end{array}$ \\
\hline $\begin{array}{c}\text { Número y } \\
\text { porcentaje } \\
\text { de actividades } \\
\text { con contexto por } \\
\text { SEA }\end{array}$ & $\begin{array}{c}n=4 \\
(13 \%)\end{array}$ & $\begin{array}{c}n=14 \\
(47 \%)\end{array}$ & $\begin{array}{c}n=3 \\
(10 \%)\end{array}$ & $\begin{array}{c}n=8 \\
(27 \%)\end{array}$ & $\begin{array}{c}n=1 \\
(13 \%)\end{array}$ & $\begin{array}{c}n=30 \\
(100 \%)\end{array}$ \\
\hline
\end{tabular}

Una vez aplicada la RIC siguiendo el ejemplo mostrado en la Tabla 3, se procedió a calcular un promedio por cada indicador de contexto en cada etapa de la estructura de cada SEA, es decir, del inicio, intermedio y final (considerando sólo las actividades contextualizadas). Posteriormente se suman los promedios de cada etapa de la SEA (inicio, medio y final) y el resultado obtenido se muestra en la Tabla 5 y la Figura 1.

Tabla 5. Promedio por cada indicador de contexto de la RIC de las cinco SEA de química de acuerdo con la estructura Inicio (I), Medio (M) y Final (F).

\begin{tabular}{|c|c|c|c|c|c|c|c|c|c|c|c|c|c|c|c|c|c|c|c|c|c|}
\hline \multirow{7}{*}{ 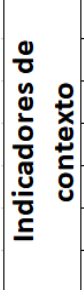 } & 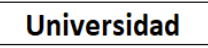 & \multicolumn{4}{|c|}{ A } & \multicolumn{4}{|c|}{ B } & \multicolumn{4}{|c|}{ C } & \multicolumn{4}{|c|}{ D } & \\
\hline & Segm & $I$ & $\mathbf{M}$ & $\mathbf{F}$ & Total & 1 & $M$ & $\mathbf{F}$ & 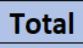 & & $M$ & $\mathbf{F}$ & . & 1 & $\mathbf{M}$ & $\mathbf{F}$ & Total & 1 & M & $\mathbf{F}$ & 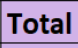 \\
\hline & Aute & 0,0 & 1,5 & 0,0 & 1 & 3,0 & 2,3 & 3,0 & & 0,0 &, 5 & 3,0 & 5 & 3,0 & 2,6 & 3,0 & 6 & 0,0 & 0,0 & 0 & 0 \\
\hline & & 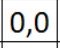 & 3 & & 1 & 0 & 1,7 & & & 0 & 1,5 & 2,0 & 3 & 2,0 & 1,6 & 2,0 & 6 & 0,0 & 0,0 & 1 & 0 \\
\hline & 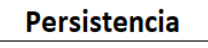 & & & & & & & & & & & & & & 4 & & & 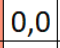 & 0 & & \\
\hline & & 0,0 & & 0,0 & & 1,0 & 1,0 & & & & 5 & 1,0 & & 1,0 & 1,8 & 2,0 & & 0,0 & 0,0 & 1,0 & 0 \\
\hline & Construcción & 0,0 & 3,0 & 0,0 & 3,0 & 3,0 & 2,1 & 1,7 & 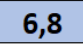 & 0,0 & 2,5 & 3,0 & 5,5 & 2,0 & 3,0 & 3,0 & 8,0 & 0,0 & 0,0 & 1,0 & 1,0 \\
\hline
\end{tabular}




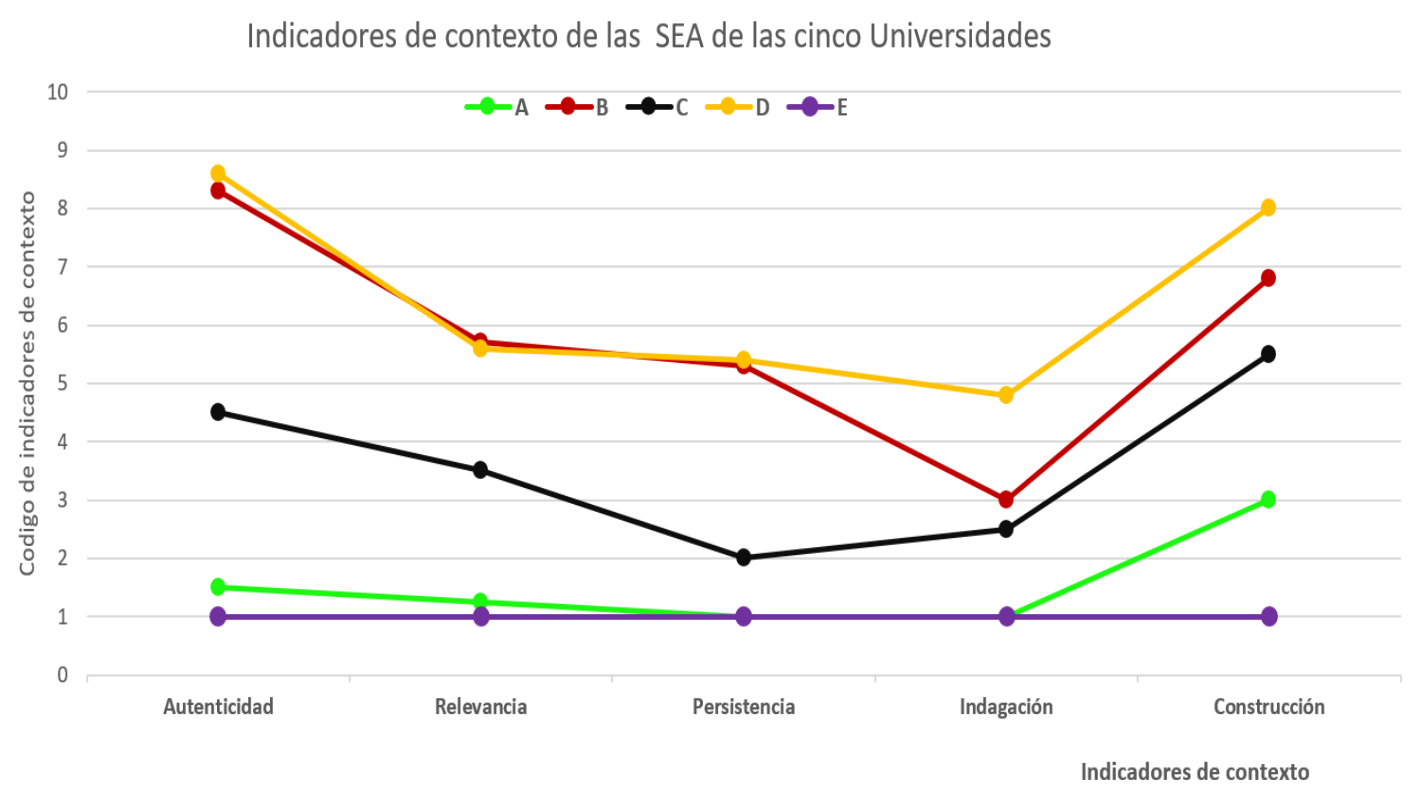

Figura 1. Indicadores de contextos para las cinco SEA de química.

A partir del análisis de la Tabla 5 y la Figura 1 se individualizan tres perfiles de contextualización, uno de perfil más alto, formado por las SEA B y D, otro de perfil medio la SEA C y finalmente un perfil más bajo formado por las SEA A y E. En el grupo de perfil contextualizador alto se encuentran las SEA caracterizadas por tener un mayor porcentaje de actividades contextualizadas (Tabla 4) y una mayor relevancia de contextos. Estos se utilizan a lo largo de toda la secuencia en sus fases inicial, medio y final, por lo que muestran una elevada persistencia. Se infiere que las actividades contextualizadas permitirán en el estudiante construir y razonar científicamente sobre un fenómeno, por lo que podemos afirmar que los contextos son mayormente constructores y en menor medida las preguntas surgidas del contexto fomentan la indagación. Dentro de este perfil destaca la SEA realizada por el profesor de formación inicial de la universidad $\mathrm{D}$, que diseña una secuencia para $1^{\circ}$ de ESO titulada La Materia con 17 actividades de las cuales 8 son contextualizadas (Tabla 4). Los títulos de todas las actividades de esta SEA muestran una diversidad, incluyendo mayoritariamente títulos conceptuales y en menor medida retóricos y contextuales. Las actividades contextualizadas de la SEA D utilizan en su mayoría contextos personales, aunque también incluyen contextos científico-tecnológico y se desarrollan a lo largo de las tres fases de la SEA. Los contextos usados en esta SEA son los mismos en las tres primeras actividades que se encuentran en la fase inicial y media de la SEA, donde se visualiza la historia de un náufrago y de un grupo de personajes que deben solucionar ciertas problemáticas presentadas. En la fase medio de la SEA se desarrollan dos contextos como todo no es magia o también se presentan fenómenos de la naturaleza. Se finaliza la SEA con un contexto nuevo pero que tiene una implicación con el contexto de la fase inicial, ya que hace mención nuevamente a los personajes que deben solucionar problemáticas que se les presentan, deben descubrir entre varios materiales el material fabricado con un 100\% de plata. Por lo tanto, la SEA D presenta contextos iguales entre actividades de la fase inicial y la fase media, pero en la fase final se vuelve a utilizar parte del contexto inicial (los personajes), en un nuevo contexto, lo que permite al estudiante mantener cierto hilo con las actividades anteriores.

El perfil contextualizador medio está representado por la SEA diseñada por la universidad C. Esta SEA está dirigida a estudiantes de $3^{\circ}$ de ESO, se titula Más allá de la física y la química y está constituida por 9 actividades, 3 de las cuales son contextualizadas. Las actividades que presentan contextos se encuentran en la fase media y final de la SEA, con contextos de tipo 
social y científico-tecnológico de un nivel medio en relación con la autenticidad, la relevancia y la persistencia. Con respecto a las preguntas diseñadas en la SEA, constatamos en primera instancia un nivel de construcción que está por encima de la media con preguntas abiertas que ayudan al estudiante a construir su modelo teórico escolar. Por ejemplo, una de las actividades en la fase media de la SEA C era debatir en relación con una situación verídica de contaminación en la piscina de un edificio de apartamentos que fue vista en video. Otra actividad que se desarrolla en la fase final consiste en el diseñar una revista cuyo objetivo es comprender el impacto a la sociedad y al medio ambiente de las reacciones químicas provocadas por la actividad bumana. Estas actividades permiten al estudiante dialogar e ir, a partir del debate y en el caso de diseñar una revista en pro del medio ambiente, hacia la búsqueda, comprensión y reelaboración de sus ideas y conocimientos que tiene hasta el momento de alguna situación en particular, en este caso acerca de las reacciones químicas.

En el perfil contextualizador bajo se sitúan las SEA A y E dirigidas a estudiantes de $3^{\circ}$ de ESO y $2^{\circ}$ de bachillerato respectivamente. Las SEA A y E están conformadas por un total de 11 y 10 actividades respectivamente de las cuales 4 y 1 son contextualizadas. Los contextos que se introducen muestran tener unos niveles de autenticidad, relevancia, y persistencia muy bajos. Mientras que las actividades contextualizadas no promueven la indagación en ninguna de las dos secuencias, el contexto de la SEA A presenta un nivel constructor mayor debido a que sus actividades contextualizadas colocan al estudiante en situaciones que lo ayudan a construir su modelo a través de preguntas como ¿Cuál será el motivo por el cual en una radiografía se ven solo los buesos y no los músculos o la piel? o ippor qué crees que hay carteles de peligro en las puertas de las salas de radiografías?. Las secuencias con un perfil contextualizador bajo están centradas en el profesor, y sus actividades consisten mayoritariamente en la presentación de un concepto, en el desarrollo de ejercicios y en algunos casos, en la comprobación de la teoría a través de una actividad experimental.

\section{Discusión y conclusiones}

Todos los profesores de ciencias en formación inicial de nuestra muestra piloto diseñaron SEA de química que utilizaban contextos en el desarrollo de las actividades. El análisis presentado pretende aportar herramientas para poder identificar y posteriormente entender las dificultades que tienen estos futuros docentes en la articulación de los contextos durante el diseño de SEA de química. El hecho de usar contextos en el diseño de SEA supone abordar la tensión entre el contexto y la construcción de conceptos y modelos de química escolar.

El análisis se ha centrado en identificar las funciones de los contextos desarrollados en las actividades de las SEA de química diseñadas por los profesores de ciencias de educación secundaria en formación inicial. Hemos construido una RIC que puede ser útil no sólo para la investigación sino también como material didáctico en la formación inicial de profesores para promover la reflexión sobre la calidad de las SEA de ciencias que diseñan. Los resultados del análisis nos han permitido organizar las 5 SEA en tres grupos correspondientes a tres perfiles contextualizadores alto, medio y bajo según el valor de la RIC aplicada a cada SEA de la muestra. Dos de los 5 indicadores de contexto incluidos en la rúbrica, Persistencia e Indagación han resultado ser siempre los más bajos en cada perfil. Esto nos indica que los profesores de ciencias en formación inicial muestran tener dificultades, por un lado, al diseñar y conectar las actividades con un contexto materializados en textos multimodales que forman parte de la SEA y por otro, en incluir propuestas de indagación conectadas con los contextos de las SEA. 


\section{a) Persistencia de los contextos a lo largo de la SEA}

Los profesores en sus diseños ponen a disposición de los estudiantes contextos como por ejemplo a) el efecto de la bumedad ambiental en una bicicleta, b) rescatar un náufrago de una isla, c) la quimica en la vida cotidiana, etc., que son contextos auténticos y relevantes, unos más que otros. Pero lo que se observa en algunos casos es que el contexto no persiste a lo largo de la secuencia. De hecho, nos encontramos con una SEA, del perfil contextualizador bajo, que tiene 9 actividades, pero sólo una de ellas presenta un contexto y se encuentra en la fase final. En este caso, tendríamos una secuencia que se ajustaría mejor al modelo de contexto tradicional apuntado por De Jong (2008). También nos encontramos con contextos de una SEA de perfil contextualizador medio, ubicados al inicio de la fase media, cuya función es captar la atención del alumnado. Pero esta "magia" sólo dura esa sesión porque luego se salta al mundo abstracto de las teorías y los símbolos, sin explicitar la necesidad de promover un proceso para que el alumnado pueda construir los conceptos científicos. En este caso tendríamos una secuencia que se adaptaría al modelo de contexto moderno propuesto por De Jong (2008). En cambio, existen dos SEA de perfil contextualizador alto en las que los contextos se van articulando a lo largo de toda la secuencia. En este caso podríamos hablar de secuencias que podrían asociarse al modelo de contexto reciente señalado por De Jong (2008).

b) Indagación a partir de contextos

De las 5 SEA analizadas en este trabajo solo una de ellas tiende a fomentar la indagación de nivel 3 conectada a un contexto de acuerdo a la RIC. Las actividades contextualizadas que fomentan la indagación de esta SEA se encuentran ubicadas en la fase final de la secuencia, donde el estudiante deberá aplicar todo el aprendizaje construido, partiendo de la formulación de una pregunta de investigación. En general las SEA de nuestra muestra hacen uso de contextos para explicar ciertos fenómenos, pero cuando se diseñan actividades experimentales éstas se basan en proponer todos los pasos y acciones que debe realizar el estudiante en el laboratorio sin referencia alguna a un contexto. Este resultado apoya los obtenidos por Bogdan Toma, Greca y Meneses-Villagrá (2017) que indican la falta de contextualización de las propuestas de indagación planteadas por futuros docentes de educación primaria.

El análisis de naturaleza exploratoria realizado en este trabajo apunta a la existencia de un problema en la formación del profesorado de ciencias de educación secundaria en relación con el uso de los contextos en la enseñanza de la química. Es un hecho el que sólo una porción pequeña de estudiantes del máster de formación del profesorado de ciencias en Catalunya diseña una SEA de enseñanza de la química durante su formación. Resulta pues urgente plantearse la necesidad de fomentar el diseño de SEA de química en las que los contextos introducidos sean más auténticos, relevantes, persistentes, constructores, e indagadores. El diseño de SEA constituyen auténticos contextos formativos en los que los docentes de ciencias de secundaria en formación inicial deben hacer explícitas sus intenciones educativas antes de ponerlas en práctica en el aula. Estas SEA actúan como hipótesis de intervención que promueven la reflexión del futuro docente con la ayuda de instrumentos como la RIC presentada en este trabajo. Estas herramientas formativas pueden facilitar la toma de decisiones del docente en formación para que pueda crear oportunidades ricas en las que los estudiantes de educación secundaria conecten los aprendizajes de química realizados en clase con las situaciones de la vida real (King et al, 2008).

\section{Agradecimientos}

Investigación parcialmente financiada por el Ministerio de Economía y Competitividad (referencia EDU201566643-C2-1-P), por la Generalitat de Catalunya (referencia 2017SGR1399) y por Beca CONICYT -Chile 2014. 


\section{Referencias}

Arriassecq, I., Greca, I. M., y Cayul, E. E. (2017). Secuencias de enseñanza y aprendizaje basadas en resultados de investigación: propuesta de un marco teórico para el abordaje de la teoría especial de la relatividad. Enseñanza de las ciencias: revista de investigación y experiencias didácticas, 35(1), 133-155.

Astudillo, C., Rivarosa, A., Ortiz, F. (2011). Formas de pensar la enseñanza en ciencias. Un análisis de secuencias didácticas. Revista Electrónica de Enseñanza de las Ciencias, 10(3), 567-586.

Blanco A., España E., Rodríguez F. (2012) Contexto y competencia científica. Alambique. Didáctica de la Ciencias Experimentales, 70, 9-18.

Blanco, A.; Franco-Mariscal, A; España, E. (2015). A Competence-based approach to the design of a teaching sequence about oral and dental health and hygiene: a case study. Journal of Biological Education, 50(2), 196-206.

Bogdan Toma, R., Greca, I. M., y Meneses-Villagrá, J. Á. (2017). Dificultades de maestros en formación inicial para diseñar unidades didácticas usando la metodología de indagación. Revista Eureka sobre Enseñanza y Divulgación de las Ciencias, 14(2), 442-457.

Broman, K. y Parchmann, I. (2014). Students' application of chemical concepts when solving chemistry problems in different contexts. Chemistry Education Research and Practice, 15(4), 516-529.

Bulte, A. M., Westbroek, H. B., de Jong, O., \& Pilot, A. (2006). A research approach to designing chemistry education using authentic practices as contexts. International Journal of Science Education, 28(9), 1063-1086.

Couso, D. (2014). La moda de "aprender indagando" a la indagación para modelizar: una reflexión crítica. XXVI Encuentro de Didáctica de las Ciencias Experimentales. Huelva (Andalucía). Recuperado de: http://uhu.es/26edce/actas/docs/conferencias/pdf/ 26ENCUENTRO_DCE-ConferenciaPlenariaInaugural.pdf

De Jong, O. (2008). Context-based chemical education: How to improve it? Chemistry Education International, 8, 1-7.

Gilbert, J. K. (2006). On the nature of "context" in chemical education. International Journal of Science Education, 28(9), 957-976.

Gilbert, J.K., Bulte, A.M.W.y Pilot, A. (2011) Concept Development and Transfer in ContextBased Science Education. International Journal of Science Education, 33(6), 817-837.

Herron, M.D., (1971). The nature of scientific inquiry, School Review, 79, 141-212.

Izquierdo M. (2006). Ciencia en contexto. Una reflexión desde la filosofía, Alambique, 46, 9-17.

Jiménez-Aleixandre, M.P. y Reigosa, C. (2006). Contextualizing practices across epistemic levels in the chemistry laboratory. Science Education, 90(4) 707-733.

King, D., Bellochi, A. y Ritchie, S. M. (2008). Making connections: Learning and teaching chemistry in context. Research in Science Education, 38(3), 365-384.

King, D.T., y Ritchie, S.M. (2012). Learning science through real-world contexts. En B. Fraser, K. Tobin y J.C. MacRobbie (Eds.), Second International Handbook of Science Education (pp. 69-80). Dordrecht, Holanda: Springer Press. 
Lupión-Cobos, T., López-Castilla, R., y Blanco-López, Á. (2017). What do science teachers think about developing scientific competences through context-based teaching? A case study. International Journal of Science Education, 39(7), 937-963.

Marchán-Carvajal, I. y Sanmartí, N. (2014). Una revisión sobre el uso de contextos en la enseñanza de las ciencias y su potencial para el desarrollo de la competencia científica. En de las Heras et. al, (coord.). Investigación y transferencia para una educación en ciencias: Un reto emocionante. (pp. 702-710). Huelva: Servicio de Publicaciones de la UHU.

Marchán-Carvajal, I., y Sanmartí, N. (2015). Criterios para el diseño de unidades didácticas contextualizadas: aplicación al aprendizaje de un modelo teórico para la estructura atómica. Educación química, 26(4), 267-274.

Méheut, M. (2005). Teaching-learning sequences tools for learning and/or research. In Research and the quality of science education (pp. 195-207). Springer, Dordrecht.

Mehéut, M. and Psillos, D. (2004). Teaching-learning sequences: aims and tools for science education research. International Journal of Science Education, 26(5), 515-535.

Parchmann, I., Grasel, C., Baer, A., Nentwig, R.D., y Ralle, B. (2006) “Chemie im Kontext”: A symbiotic implementation of a context-based teaching and Learning approach, International Journal of Science Education, 28(9), 1041-1062.

Podschuweit, S. y Bernholt, S. (2018). Composition-Effects of Context-based Learning Opportunities on Students' Understanding of Energy. Research in Science Education, 48,717-752.

Prins, G. T., Bulte, A. M. W., Van Driel, J. H., y Pilot, A. (2008). Selection of authentic modelling practices as contexts for chemistry education. International Journal of Science Education, 30(14), 1867-1890.

Pilot, A. y Bulte, A. (2006b). The use of "contexts" as a challenge for the chemistry curriculum: Its successes and the need for further development and understanding. International Journal of Science Education, 28(9),1087-1112.

Rodríguez, F y Blanco, Á (2015). ¿Por qué bebemos agua embotellada? Una propuesta para la enseñanza de la física y química en $3^{\circ}$ de la ESO. En Blanco, A; Lupión, T. (ed.). La competencia científica en las aulas: Nueve propuestas didácticas. (pp.205-44). Santiago de Compostela: Andavira Editora.

Roca, M., Marquez, C. y Sanmartí, N. (2013) Las preguntas de los alumnos: Una propuesta de análisis. Enseñanza de Las Ciencias, 31(1), 95-114.

Sanmartí, N. y Márquez, C. (2017). Aprendizaje de las ciencias basado en proyectos: del contexto a la acción. Apice. Revista de Educación científica, 1(1), 3-16.

Stuckey, M., Hofstein, A., Mamlok-Naaman, R., y Eilks, I. (2013). The meaning of 'relevance' in science education and its implications for the science currículum, Studies in Science Education, 49(1), 1-34.

Tamir, P., y García, M. P. (1992). Características de los ejercicios de prácticas de laboratorio incluidos en los libros de textos de Ciencias utilizados en Cataluña. Enseñanza de las ciencias: revista de investigación y experiencias didácticas, 10(1), 3-12.

Weber, R. P. (1990). Basic Content Analysis, 2nd ed. Newbury Park, CA.: Sage Publications. 